\title{
O tratamento farmacológico do transtorno bipolar na infância e adolescência
} Pharmacological Treatment of Juvenile Bipolar Disorder

\author{
Luis Augusto RoHdE ${ }^{1}$ \\ SiLZÁ TRAMONTINA ${ }^{2}$
}

\begin{abstract}
Resumo
O reconhecimento do transtorno do humor bipolar (THB) em crianças e adolescentes tem aumentado significativamente nos últimos anos. O THB, nessa faixa etária, parece freqüentemente se apresentar de forma atípica, assim, humor irritável com "tempestades afetivas" são mais freqüentes do que euforia, o curso da doença é mais crônico do que episódico e sintomas mistos com depressão e mania concomitantes são comuns. Alta prevalência de comorbidades, em especial com transtorno do déficit de atenção/hiperatividade, parece ser a regra. Apesar do efeito devastador do THB no desenvolvimento infantil, poucos estudos têm investigado intervenções farmacológicas nesses pacientes. Essa revisão tem como objetivo apresentar uma discussão crítica dos achados provenientes de estudos recentes nessa nova área de pesquisa, a psicofarmacologia do THB em crianças e adolescentes. Para realizar essa tarefa, uma revisão computadorizada e sistemática da literatura foi realizada por meio do PUBMED. Os dados sobre tratamento psicofarmacológico do THB em crianças e adolescentes são apresentados em três seções: 1) a força da evidência científica na área; 2) descrição crítica dos estudos principais; 3) proposição de um algoritmo de decisão. Apenas um estudo randomizado duplo-cego e controlado por placebo foi encontrado. A quase totalidade dos estudos é composta de ensaios prospectivos abertos, séries de casos e análises retrospectivas de prontuários. Os fármacos mais estudados são o lítio e o valproato de sódio. Essa revisão indica uma escassa disponibilidade de evidência científica de qualidade para guiar o clínico na decisão do tratamento farmacológico a ser indicado para o THB em crianças e adolescentes.
\end{abstract}

Palavras-chave: Crianças, adolescentes, mania, transtorno do humor, tratamento, farmacologia.

1 Professor Adjunto de Psiquiatria da Infância e Adolescência da Universidade Federal do Rio Grande do Sul - UFRGS.

2 Médica contratada do Serviço de Psiquiatria da Infância e Adolescência do Hospital de Clínicas de Porto Alegre. Mestre em Medicina e Doutoranda em Psiquiatria - UFRGS.

Endereço para correspondência: Luis Augusto Rohde. Serviço de Psiquiatria da Infância e Adolescência do Hospital de Clínicas de Porto Alegre. Rua Ramiro Barcelos, 2350 - $4^{\circ}$ andar 90035-003 - Porto Alegre - RS; e-mail: lrohde@terra.com.br 


\begin{abstract}
Juvenile Bipolar Disorder (JBD) has been recognized more frequently in the last years. The disorder might have an atypical presentation in this age range. Thus, irritability with "affective storms" are more frequent than euphoria, the evolution of the disorder is more chronic than episodic and mixed symptoms are more frequent. High prevalence of comorbid conditions, specially AttentionDeficit/Hyperactivity Disorder, seems to be the rule. Despite the devastating effect of JBD in the child development, few pharmacological investigations were conducted in these patients. This review aims to present a critical discussion of the findings from this emerging new area of research, the psychopharmacology of the JBD. To accomplish this task, a systematic computerized search of the literature was conducted through the PUBMED. Findings are presented in three sections: 1) the strength of the scientific evidence in the field; 2) critical description of the main investigations; 3 ) proposition of an algorithm to guide treatment options. Only one randomized, double-blind, controlled trial was found in the literature. Almost all studies are open prospective trials, case series, or retrospective analyses of medical records. The most investigated drugs are lithium and valproate sodium. This review suggest that a scarce availability of high quality evidence to guide clinicians in the decision on which pharmacological treatment should be used to address bipolar disorder in children and adolescents.
\end{abstract}

Key words: Children, adolescents, mania, mood disorders, treatment, pharmacology.

\section{Introdução}

A maioria dos estudos sobre transtorno de humor bipolar (THB) em crianças e adolescentes foi realizada nos Estados Unidos e Canadá. Poucos estudos epidemiológicos têm examinado a prevalência e/ou a incidência do THB em crianças e adolescentes. Lewinsohn et al. (1995) encontraram uma prevalência ao longo da vida do transtorno bipolar tipo I ou II em torno de $1 \%$ em uma amostra de escolares de idade entre 14 e 18 anos. Recentemente, em um estudo no ambulatório de psicofarmacologia do Serviço de Psiquiatria da Infância e Adolescência do HCPA, identificou'se uma prevalência de THB em jovens com menos de 15 anos ao redor de 7,2\% (amostra clínica) (Tramontina et al., 2003).

Há controvérsias em torno dos sintomas apresentados por crianças e adolescentes e o correto diagnóstico do THB nesta faixa etária. Sintomas de mania em adolescentes podem-se apresentar de forma diferente dos sintomas dos adultos com maior prevalência de sintomatologia mista e psicose. Transtorno bipolar prépuberal parece freqüentemente se apresentar de forma atípica; humor irritável com "tempestades afetivas" são mais freqüentes do que euforia, o curso da doença é mais crônico do que episódico e sintomas mistos com depressão e mania concomitantes caracterizam o THB em crianças e adolescentes mais jovens. Além disso, ciclagem rápida, ultra-rápida e dentro do mesmo dia podem ser encontradas mais freqüentemente nesses pacientes (para uma revisão aprofundada ver Leiben- luft et al., 2003). Esses autores propuseram que o THB em crianças e adolescentes pudesse ser dividido em quatro diferentes grupos baseados na apresentação fenotípica: 1) pacientes com sintomatologia completa para o diagnóstico de THB de acordo com o DSM-IV com presença de grandiosidade e/ou euforia (fenótipo mais estreito);2) pacientes com sintomatologia maníaca e episódios claros de acordo com o DSM-IV, entretanto com duração de episódios entre 1 e 3 dias (fenótipo intermediário); 3) pacientes com episódios claros de irritabilidade e sintomas de mania (mas não euforia) (fenótipo intermediário); 4) pacientes com sintomatologia crônica (não-episódica) que não inclui os sintomas típicos de mania, mas compartilha a grave irritabilidade com hiperexcitabilidade com os fenótipos mais estreitos (fenótipo mais amplo).

Outro importante aspecto a ser levado em conta no diagnóstico do THB na população pediátrica é a alta prevalência de comorbidade. Altas taxas de comorbidade com transtorno de déficit de atenção/ hiperatividade (TDAH) (mais de 75\%) são encontradas em amostras clínicas de crianças e adolescentes com THB e altas taxas (10\% a 15\%) de THB também são achadas em muitas amostras clínicas de crianças com TDAH (Pavuluri et al., 2002). Em virtude da existência dessas altas taxas de comorbidade entre TDAH e THB, alguns investigadores sugerem que a presença de sintomas do TDAH, em crianças que mais tarde apresentarão diagnóstico de THB, pode definir um subtipo específico de THB no qual o TDAH aparece 
como um pródromo do transtorno de humor (Strober et al., 1988). Altas taxas de comorbidade com outros transtornos, como os do comportamento disruptivo e ansiedade, também são evidenciadas em jovens pacientes bipolares (Tramontina et al., 2003).

É importante salientar que, apesar de existirem incertezas no diagnóstico do transtorno de humor bipolar (THB) em crianças e adolescentes, este transtorno afeta seriamente o desenvolvimento e o crescimento emocional desses pacientes. Ele está associado com altas taxas de tentativa de suicídio, dificuldades escolares, comportamento de alto risco como promiscuidade sexual e abuso de substâncias, dificuldades nas relações interpessoais, problemas legais e múltiplas hospitalizações (Pavuluri et al., 2002). Apesar do efeito devastador do THB no desenvolvimento infantil, poucos estudos têm investigado intervenções farmacológicas nesses pacientes.

Esta revisão tem como objetivo apresentar uma discussão crítica dos achados provenientes de estudos recentes nessa nova área de pesquisa, a psicofarmacologia do THB em crianças e adolescentes. Para realizar essa tarefa, uma revisão computadorizada e sistemática da literatura foi realizada por meio do PUBMED. As referências foram procuradas utilizando a seguinte estratégia: Bipolar Disorder [Título/ Resumo] AND (Children OR Adolesc* [Título/Resumo]) AND (Medication OR Psychopharmac* [Título/ Resumo]). Todos os resumos em inglês $(n=295)$ foram considerados na revisão. As referências dos artigos significativos foram revisadas. Por fim, como o primeiro autor (LAR) é membro de reunião anual do National Institute of Mental Health sobre THB em crianças e adolescentes, que congrega os pesquisadores da área, dados não publicados sobre o tema foram solicitados para eles. Os dados sobre tratamento psicofarmacológico do THB em crianças e adolescentes são apresentados em três seções: 1) a força da evidência científica na área; 2) descrição crítica dos principais estudos; 3) proposição de um algoritmo de decisão.

\section{Apresentação e discussão}

\section{A força da evidência científica na área}

O primeiro aspecto que chama atenção numa revisão mais cuidadosa sobre o tema é a falta de estudos considerados de nível A (ensaios clínicos controlados, randomizados, duplos-cegos). Como resultado da presente revisão, encontra-se apenas um artigo com tal delineamento, e a amostra era específica para THB em comorbidade com transtorno de uso de substâncias (Geller et al., 1998). Assim, em recente reunião de consenso sobre as necessidades não preenchidas no diagnóstico e tratamento dos transtornos de humor em crianças e adolescentes ressaltou-se que, embora exista uma crescente disponibilidade de estudos sobre o tratamento farmacológico do THB juvenil, estes consistem quase exclusivamente de relatos ou séries de casos, estudos abertos e investigações sem critérios padronizados. Assim, tratamentos baseados em evidência de nível A ainda não podem ser preconizados para o THB juvenil (Coyle et al., 2003). Nesse sentido, em uma outra reunião de consenso entre investigadores discutiram-se as questões e os aspectos controversos na metodologia de ensaios clínicos em crianças e adolescentes com THB. Foram apresentadas sugestões em relação a possíveis delineamentos de pesquisa. Uma das principais recomendações foi a necessidade de que as agências governamentais financiem ensaios clínicos controlados por placebo em crianças e adolescentes de 10 a 17 anos com episódios agudos de mania com ou sem comorbidades (para uma revisão ver Carlson et al., 2003).

\section{Descrição crítica dos principais estudos}

Tomando como base as questões mencionadas, pode-se dividir essa seção em três grupos: 1) o único ensaio clínico controlado por placebo; 2) séries de caso ou estudos abertos; 3) séries de caso ou estudos abertos com amostras específicas ou que respondem a questões específicas. Nessa seção, somente foram considerados aqueles estudos que apresentavam confiabilidade diagnóstica e uso de escalas padronizadas para avaliação da evolução (mesmo que não fossem específicas para THB, como, por exemplo, escalas de avaliação de funcionamento global). Além disso, foram incluídos apenas estudos com tamanho amostral superior a dez casos e que avaliaram amostras exclusivas de crianças e adolescentes.

\section{0 único ensaio clínico controlado por placebo}

Geller et al. (1998) conduziram um ensaio clínico com grupos em paralelo controlado por placebo, duplo-cego, avaliando a eficácia do lítio em 25 adolescentes (idade: $16,3 \pm 1,2$ anos) com THB e transtorno por uso de substâncias durante seis semanas. A evolução dos sintomas maníacos foi estimada usando o Children Global Assessment Scale (CGAS), que avalia o funcionamento global do indivíduo. $\mathrm{O}$ uso de drogas foi avaliado por dosagens urinárias semanais, mas com solicitação randomizada. O tratamento com lítio se associou a uma melhora significativamente mais pronunciada tanto no funcionamento global como no uso de drogas, embora vieses de aferição possam ter favorecido o tratamento ativo na questão do uso de drogas (Szobot e Rohde, 1999). Além disso, o diagnóstico de THB foi feito de forma flexível, permitindo a entrada no protocolo de pacientes com quadros de depressão maior e preditores de bipolaridade.

\section{Séries de caso ou estudos abertos com amostras de pacientes com THB juvenil}

A apresentação dos estudos nessa subseção obedece a uma hierarquização de acordo com os seguintes critérios: 1) número de medicações avaliadas (priori- 
dade para monoterapia sobre polifarmacoterapia);2) tipo de delineamento em relação ao eixo de montagem do estudo (prioridade para estudos prospectivos sobre retrospectivos); 3) delineamento (prioridade para estudos comparativos - desde que randomizados sobre estudos com uma única medicação); 4) tamanho amostral (prioridade para os estudos com maior tamanho amostral).

Um estudo aberto randomizado e em paralelo com 42 crianças e adolescentes com THB juvenil dos tipos I e II avaliou a eficácia do lítio, do divalproato de sódio e da carbamazepina em monoterapia durante seis semanas. As medidas primárias de eficácia foram baseadas numa escala específica para mania (Young Mania Rating Scale - YMRS) e numa escala de avaliação de funcionamento global (Clinical Global Impression - CGI). Tomando como base a diferença entre os escores basais e os finais na YMRS, todas as três medicações apresentaram tamanho de efeito considerado na faixa excelente $(>0,80)$. Não foram detectadas diferenças significativas entre as três medicações, embora o estudo talvez não tenha tido poder suficiente para detectar potenciais diferenças (Kowatch et al., 2000). Em relação a eventos adversos, náusea foi o sintoma mais freqüente nos três grupos, seguido de sedação, que ocorreu em $15 \%$ dos pacientes que recebiam carbamazepina e $20 \%$ dos pacientes em uso de divalproato de sódio. Aumento de apetite aconteceu apenas no grupo que recebia lítio (14\%). A maioria dos eventos adversos foi considerada de intensidade mínima à moderada, sendo tolerados pela maioria dos pacientes.

Kafantaris et al. (2003) conduziram um estudo aberto de quatro semanas com 100 adolescentes de 12 a 18 anos, que estavam em episódio maníaco agudo como parte do transtorno do humor bipolar tipo I para avaliar a eficácia do lítio. As definições de melhora e remissão foram claramente estabelecidas e baseadas numa redução clinicamente significativa na YMRS e na CGI, como no estudo anterior. Foram detectadas diferenças estatisticamente significativas entre os escores basais e finais nas escalas mencionadas, sendo grande o tamanho de efeito $(>0,80)$. Enquanto 63\% dos pacientes preencheram critérios para melhora, somente $26 \%$ foram considerados com remissão completa dos sintomas maníacos. Aumento de peso foi o evento adverso mais freqüente. $\mathrm{O}$ uso de antipsicóticos como agentes coadjuvantes nos pacientes com sintomatologia psicótica implicou redução sintomatológica neles de igual tamanho do que aquela verificada nos pacientes sem sintomatologia psicótica em monoterapia com lítio.

Na mesma direção, Strober et al. (1988) documentaram uma razoável taxa de melhora com o lítio num estudo aberto com 50 adolescentes com THB tipo I, tomando como base uma medida de funcionamento global ( $56 \%$ após quatro semanas e $68 \%$ após seis semanas).
Wagner et al. (2002) realizaram um estudo aberto de até oito semanas de duração com divalproato de sódio em 36 crianças e adolescentes com THB. Nesse protocolo, foram incluídos pacientes em episódios maníacos, hipomaníacos ou mistos. Amedida primária de eficácia foi a Mania Rating Scale (MRS). Uma diferença significativa foi detectada entre o escore basal e o final na MRS, determinando um tamanho de efeito igual a 1,12 . Vinte e dois pacientes (61\%) dos 36 apresentaram reduções $\geq 50 \%$ na MRS. A interpretação desse estudo é muito difícil, já que mais da metade dos pacientes descontinuou prematuramente o protocolo e pelo menos $47 \%$ dos participantes utilizaram outras medicações durante o estudo, tais como estimulantes, haloperidol, e lorazepam. O protocolo permitia a associação com lítio nos pacientes que não respondessem adequadamente ao divalproato de sódio até o $35^{\circ}$ dia. Vinte e sete pacientes $(68 \%)$ relataram um ou mais eventos adversos durante o estudo, sendo os mais comuns cefaléia (18\%) e náuseas (18\%). Seis pacientes descontinuaram o protocolo prematuramente por eventos adversos. Em pelo menos dois pacientes com rash cutâneo julgou-se clinicamente alta a probabilidade de relação do evento com o uso da medicação.

Frazier et al. (2001) demonstraram a eficácia da olanzapina na redução de sintomatologia maníaca num estudo aberto de oito semanas com 23 crianças e adolescentes de 5 a 14 anos com THB. Nesse protocolo, foram incluídos pacientes em episódios maníacos, hipomaníacos ou mistos. Os sintomas foram avaliados pela YMRS e pela subescala de severidade da CGI, entre outras. A quase totalidade dos pacientes $(n=22)$ completou o protocolo. Uma redução significativa dos escores basais da YMRS foi verificada com a medicação no final do estudo. Definindo melhora como uma redução de pelo menos $30 \%$ dos sintomas na YMRS e um escore $\leq 3$ na subescala de severidade da CGI, $61 \%$ atingiram ou ultrapassaram esse limiar. Aumento significativo de peso foi o principal evento adverso nesse protocolo.

Em um estudo aberto, 11 crianças e adolescentes com THB foram tratados por um ano com carbamazepina. A medida de eficácia foi definida como manutenção de humor eutímico no período. Sete pacientes (64\%) foram definidos como respondedores, ou seja, não apresentaram episódios depressivos, maníacos ou mistos no período. Não foram registrados eventos adversos clinicamente significativos (não houve necessidade de interrupção do tratamento em nenhum caso) (Bouvard et.al, 1993).

Vários estudos retrospectivos por meio de exame de prontuários foram conduzidos, sugerindo a eficácia do lítio, da risperidona e do divalproato de sódio no controle de sintomas maníacos em crianças e adolescentes com THB (Davanzo et al., 2003; Henry et al., 2003; Frazier et al., 1999). Todos utilizaram uma metodologia de estimar, a partir de análise de prontuários, 
escores basais e na última consulta, ou durante o tratamento na CGI. Por exemplo, Davanzo et al. (2003) avaliaram prontuários de 44 pré-adolescentes com diagnóstico clínico de THB durante a hospitalização. Compararam a melhora clínica entre os pacientes que receberam lítio, divalproato de sódio e carbamazepina por meio da ANOVA para medidas repetidas, tomando como base escores na CGI para cada consulta que eram estimados por quatro clínicos com avaliação de confiabilidade interentrevistadores. Esses clínicos avaliaram independentemente os prontuários sem saber qual medicação havia sido prescrita e o escore médio deles para cada consulta era considerado na análise. A partir da segunda semana, uma redução significativamente maior ocorreu nos grupos recebendo lítio e divalproato de sódio do que naquele que recebeu carbamazepina. Além de todos os vieses associados a estudos retrospectivos, boa parte dos pacientes nesses estudos recebeu várias outras medicações concomitantemente e a avaliação do diagnóstico foi baseada apenas na impressão clínica do médico assistente.

A maioria das crianças e adolescentes com THB não responde apenas a uma medicação, sendo necessário o uso de polifarmacoterapia. Assim, em revisão retrospectiva de prontuários na unidade de psicofarmacologia da infância e adolescência do HCPA, apenas $22 \%$ dos pacientes em monoterapia, seja com estabilizadores do humor ou antipsicóticos, atingiram uma redução $\geq$ $50 \%$ da sintomatologia (Tramontina et al., 2003). Em um estudo aberto de seguimento por quatro meses, Kowatch et al. (2003) reavaliaram 35 pacientes que haviam finalizado seu estudo aberto de seis semanas com carbamazepina, divalproato de sódio ou lítio (Kowatch et al., 2000 - ver acima). Quinze pacientes (42\%) puderam ser mantidos durante a fase de seguimento em monoterapia. A taxa de resposta ao final da fase de seguimento nesse grupo foi de $94 \%$. Entretanto, 20 pacientes (58\%) tiveram de ser mantidos em polifarmacoterapia durante o seguimento. Para esses, a taxa de resposta foi de $80 \%$. As combinações mais freqüentes foram: dois estabilizadores do humor + estimulante, dois estabilizadores do humor + antipsicótico, ou dois estabilizadores do humor + antidepressivo. Bhangoo et al. (2003) avaliaram, por triagem telefônica, famílias de crianças e adolescentes que haviam recebido o diagnóstico de THB pelos seus psiquiatras e que estavam procurando informações para se engajar em pesquisa do National Institute of Mental Health dos EUA sobre o assunto. O número médio de medicações em uso corrente foi de 3,4 , sendo os estabilizadores do humor mais utilizados o divalproato de sódio e o lítio.

Num estudo de seis semanas controlado, em paralelo, randomizado e duplo-cego, DelBello et al. (2003) avaliaram a eficácia do divalproato de sódio em associação com quetiapina contra divalproato de sódio em associação com placebo em 30 adolescentes hospitalizados com THB do tipo I, que estavam num episódio corrente maníaco ou misto. A medida primária de eficácia foi a variação do escore na YMRS entre a linha basal e o momento final do estudo. Ambos os grupos apresentaram reduções significativas nos escores da YMRS entre a linha basal e a final; entretanto, a redução foi significativamente maior no grupo que recebeu divalproato de sódio em associação com quetiapina. Definindo resposta como uma redução $\geq 50 \%$ dos escores na YMRS, significativamente mais pacientes no grupo do divalproato de sódio + quetiapina (87\%) atingiram ou ultrapassaram esse limiar do que pacientes no grupo do divalproato de sódio + placebo (53\%). Os eventos adversos foram considerados na faixa de leve a moderado em ambos os grupos pelos pacientes e suas famílias. Os eventos adversos mais comuns foram: sedação, náusea, cefaléia e irritação gastrointestinal. Apenas sedação foi significativamente mais freqüente no grupo do divalproato de sódio + quetiapina. Além da falta de um braço somente com placebo, outra limitação importante desse estudo foi a retirada inicial dos pacientes não-respondedores ao divalproato de sódio.

Findling et al. (2003) conduziram um estudo aberto de duração de até 20 semanas com 90 crianças e adolescentes (5 a 17 anos) apresentando THB dos tipos I ou II ( $81 \%$ com episódio corrente misto ou maníaco) para avaliar a eficácia da associação de lítio com o divalproato de sódio. Escalas padronizadas foram utilizadas para avaliar a evolução (YMRS, Clinical Global Assessment Scale - CGAS e Children Depression Rating Scale - Revised - CDRS-R). Reduções significativas foram encontradas em todas as escalas já a partir da oitava semana. Definindo remissão como escores na YMRS $\leq 12,5$ + escores na CDRS-R $\leq 40+$ escores na CGAS $\geq 51,47 \%$ dos pacientes atingiram ou ultrapassaram esse limiar. Detectou-se uma prevalência de sintomatologia psicótica significativamente maior nos pacientes que não tiveram remissão devida à persistência de sintomatologia psiquiátrica do que naqueles que foram considerados como apresentando remissão, sugerindo a necessidade de tratamento específico com antipsicóticos na vigência de sintomatologia psicótica acompanhando o THB. Quinze pacientes $(16,7 \%)$ descontinuaram o protocolo por eventos adversos, dos quais os mais freqüentes foram vômito, enurese, dor abdominal, tremor e sede aumentada. Um aumento significativo de peso foi detectado entre a avaliação basal e a final do estudo (aumento médio de $0,3 \mathrm{~kg} / \mathrm{semana}$ nas primeiras oito semanas e $0,2 \mathrm{~kg} /$ semana a partir da oitava semana). Como em outros ensaios abertos, mais da metade dos pacientes nesse protocolo estava recebendo outras medicações, principalmente estimulantes $(52,2 \%)$ e antipsicóticos $(14,4 \%)$, tornando difícil a interpretação dos resultados. Gracious et al. (2004) reavaliaram os níveis do hormônio estimulante da tireóide (TSH) nos pacientes desse estudo, documentando que $24,4 \%$ deles tinham aumento dos níveis do TSH durante o protocolo 
atingindo níveis $\geq 10 \mathrm{mU} / \mathrm{L}$ no final do protocolo. Uma associação entre níveis séricos do TSH e níveis séricos do lítio durante o protocolo foi encontrada. Esses dados sugerem a necessidade de verificação de função da tireóide em crianças e adolescentes recebendo lítio.

Kafantaris et al. (2001) realizaram uma análise interina no seu estudo aberto para avaliar a eficácia do lítio. Para essa análise, consideraram apenas os pacientes alocados até aquele momento com diagnóstico de THB e presença de sintomatologia psicótica. Foram incluídos adolescentes de 12 a 18 anos em episódio corrente maníaco ou de sintomatologia mista. Trinta e cinco pacientes foram elegíveis para tratamento combinado com lítio e antipsicótico por quatro semanas. Desses, 28 (80\%) completaram o protocolo. Houve uma diminuição significativa dos sintomas entre a linha basal e a quarta semana em todas as escalas avaliadas (incluindo YMRS e CGI). De acordo com a prática clínica padrão (retirada do antipsicótico após estabilização do quadro), julgou-se clinicamente que apenas 14 pacientes poderiam permanecer sem 0 antipsicótico após as quatro semanas. Desses, oito permaneceram estáveis por mais quatro semanas em monoterapia com o lítio e seis apresentaram exacerbação dos sintomas com a retirada do antipsicótico. Os seguintes fatores associaram-se com manutenção da estabilização do quadro mesmo em monoterapia: ser o primeiro episódio psicótico, menor duração do episódio psicótico corrente e presença de transtorno do pensamento em comorbidade. Esses resultados sugeriram que é possível uma estabilização rápida dos quadros bipolares com a presença de sintomas psicóticos em adolescentes com o uso combinado de lítio e antipsicóticos, mas a retirada do antipsicótico, mesmo após estabilização, associa-se freqüentemente com piora significativa da sintomatologia.

West et al. (1994) adicionaram divalproato ao tratamento de 11 adolescentes que estavam em episódio maníaco, mas não tinham tido resposta adequada ao lítio ou aos antipsicóticos. A maioria desses pacientes teve uma melhora pelo menos moderada com a adição do divalproato ao seu regime medicamentoso.

DelBello et al. (2002), em estudo retrospectivo por meio de análise de prontuários que incluiu 26 pacientes com THB entre 5 e 20 anos, documentaram que a associação de topiramato ao tratamento em curso (em geral, estabilizador do humor e/ou antipsicóticos atípicos) determinou uma diminuição significativa nos escores da CGI (tamanho de efeito = 2,6). Assim, 69\% dos pacientes foram considerados respondedores (CGI $\leq 2$ ) em algum momento do estudo após a inclusão do topiramato no regime medicamentoso. Alterações cognitivas foram os eventos adversos mais encontrados. Uma redução significativa de peso foi encontrada entre o momento de introdução do topiramato e a avaliação final.
Séries de caso ou estudos abertos com amostras específicas ou que respondem a questões específicas

A) Evidência da PRECipitaÇÃo de Mania COM o uso DE METILFENIDATO EM INDIVÍDUOS COM THB OU EM RISCO PARA O TRANSTORNO

Carlson et al. (2000) avaliaram longitudinalmente uma amostra de 75 crianças de 6 a 12 anos com TDAH e diversas comorbidades. Na idade adulta (21 a 23 anos), aqueles com história de diagnóstico de THB (mania, hipomania ou ciclotimia na idade adulta) não diferiram em qualquer aspecto relacionado ao uso prévio de metilfenidato daqueles sem história de diagnóstico de THB ao longo da vida. Esses dados não sugeriram que o metilfenidato precipitasse THB em jovens suscetíveis. Entretanto, esse estudo talvez não tivesse poder para avaliar a questão de pesquisa e o desenho ideal (comparação do grupo de risco com e sem tratamento com metilfenidato) para responder essa questão não foi empregado. Em um estudo retrospectivo e baseado apenas no diagnóstico clínico (sem uso de instrumento de diagnóstico estruturado) com 80 adolescentes com THB hospitalizados (episódio maníaco ou misto), Soutullo et al. (2002) constataram que o uso prévio de metilfenidato associou-se a um curso de doença mais grave não explicado pela comorbidade com TDAH. Portanto, essa ainda é uma questão de pesquisa em aberto.

B) Tratamento DA Depressão BIPOLAR EM CRIANÇAS E ADOLESCENTES

Biederman et al. (2000) realizaram um estudo retrospectivo de análise de prontuários de 59 pacientes com THB. O uso de inibidores seletivos da recaptura de serotonina (ISRS) associou-se à melhora significativa de depressão bipolar. Entretanto, também se verificou um aumento da chance de recorrência de sintomatologia maníaca com o uso dessas medicações. $\mathrm{O}$ uso de estabilizadores do humor, embora melhorando os sintomas maníacos, não teve efeito na depressão bipolar. Os ISRS não interferiram nas propriedades de melhora dos sintomas maníacos dos estabilizadores do humor. Assim, esse estudo sugere que o tratamento da depressão bipolar em crianças e adolescentes possa ser feito com ISRS, mas que o uso dessas medicações possa ser antecedido de adequado controle dos sintomas maníacos com estabilizadores do humor.

C) Evidência associando TDAH E PIOR REsposta A ESTABILIZADORES DO HUMOR EM THB DE CRIANÇA E ADOLESCENTES

Strober et al. (1998) realizaram um estudo aberto comparativo com o uso de lítio durante 28 dias em dois grupos de adolescentes ( 13 a 17 anos) com mania; um grupo com diagnóstico prévio de TDAH e outro pareado por sexo e idade, mas sem o transtorno. Escalas específicas para mania e para funcionamento global foram utilizadas. A melhora dos sintomas maníacos 
foi significativamente maior no grupo com THB sem diagnóstico prévio de TDAH. Entretanto, Kafantaris et al. (2003), em estudo mencionado acima, não encontraram efeito preditivo do diagnóstico de TDAH na resposta ao lítio em adolescentes com THB do tipo I. Portanto, essa é outra área de pesquisa em aberto.

D) THB EM CRIANÇAS PRÉ-ESCOLARES: AVALIAÇÃO DA INTERVENÇÃO FARMACOLÓGICA

Biederman (2004) avaliou 38 crianças em idade préescolar (4 a 6 anos) com diagnóstico provável de THB em um protocolo aberto de oito semanas. Os pacientes foram alocados para risperidona (dose média $=1,5 \mathrm{mg} /$ dia $\pm 0,5 \mathrm{mg} / \mathrm{dia})$ ou olanzapina $(6,5 \mathrm{mg} / \mathrm{dia} \pm 2,6 \mathrm{mg} /$ dia). As características basais dos pacientes nos dois grupos eram similares. Diferenças significativas nos escores da YMRS foram encontradas com ambas as medicações ao longo das oito semanas; entretanto, não se detectaram diferenças significativas entre os grupos nem ao longo das oito semanas, nem quando apenas os escores no final do protocolo foram comparados entre os grupos. Sintomas residuais significativos foram detectados em ambos os grupos. A taxa de saída do estudo foi significativamente maior com a olanzapina do que com a risperidona. Ambos os grupos tiveram aumento significativo de prolactina. Detectou-se tendência para um maior ganho de peso com a olanzapina do que com a risperidona.

\section{Proposição de um algoritmo para tratamento de THB em crianças e adolescentes}

Pelo exposto anteriormente, fica evidente que a construção de um algoritmo de decisão para o tratamento farmacológico do THB em crianças e adolescentes se baseia muito mais em estudos abertos, retrospectivos, séries de casos, ou em consensos clínicos de experts. Portanto, não existe ainda evidência de qualidade para a construção de um algoritmo sólido na área. A presente proposição é uma adaptação de um algoritmo construído a partir de consenso de investigadores (Pavuluri et al., 2002) (Figura 1). Recentemente, Pavuluri et al. (2004a) demonstraram a factibilidade da aplicação desse algoritmo em um serviço universitário, bem como indicativos de uma melhor evolução nos pacientes que seguiram o protocolo em relação aos que seguiram o tratamento convencional adotado pelo seu médico.

\section{$1^{\circ}$ passo}

O ponto de partida sempre é uma adequada avaliação diagnóstica com especial cuidado na definição do tipo de THB que a criança/adolescente apresente (THB tipo I ou II; ou ainda com fenótipo abrangente = nãoespecificado). Além disso, é importante uma extensiva pesquisa clínica das comorbidades potencialmente presentes. Por fim, é importante uma determinação mais objetiva da intensidade dos sintomas do paciente. Isso pode ser feito com o uso de escalas objetivas (vide acima) ou em ambiente clínico, de forma simplificada, com o uso de uma escala visual análoga de 0 a 10 (pede-se ao paciente que pontue a intensidade dos sintomas em cada consulta).A relevância clínica dessa avaliação reside no fato de que, em psicofarmacologia na infância e adolescência, é necessário tratar os sintomas com uma estratégia de aumento de dose até não haver mais espaço para melhora, ou a presença de eventos adversos que impeçam o aumento de dose.

\section{$2^{\circ}$ passo: THB tipo I ou II}

Iniciar com um estabilizador de humor. Embora não existam estudos comparativos bem conduzidos metodologicamente na literatura, a primeira escolha recai sobre o lítio (atingir doses superiores a $30 \mathrm{mg} / \mathrm{kg} / \mathrm{dia}$; nível sérico: até $1,2 \mathrm{mEq} / \mathrm{L}$ ), ou divalproato de sódio (atingir doses superiores a $20 \mathrm{mg} / \mathrm{kg} /$ dia; nível sérico: até $130 \mu \mathrm{g} / \mathrm{L}$ ) em função do maior número de pacientes tratados nos estudos. Outra opção menos estudada, mas com indicativos de resposta é a carbamazepina (atingir doses superiores a $15 \mathrm{mg} / \mathrm{kg} /$ dia; nível sérico entre 7 e $12 \mu \mathrm{g} / \mathrm{L})$. Outras opções, como lamotrigina ou gabapentina, são muito pouco estudadas em crianças (só relato de casos).

Diante da ausência de resposta com doses adequadas (menos de 10\% de melhora) ou resposta parcial (melhora $>10 \% \mathrm{e}<50 \%$ ) as estratégias possíveis são: 1) adicionar um antipsicótico. A primeira escolha recai sobre a quetiapina, já que o único estudo controlado foi realizado com essa medicação (atingir doses ao redor de $450 \mathrm{mg} / \mathrm{dia}$ ), mas outros antipsicóticos de segunda geração como a risperidona (até $6 \mathrm{mg} / \mathrm{dia}$ ) e a olanzapina (até $20 \mathrm{mg} /$ dia) também podem se utilizados; 2) adicionar outro estabilizador do humor (se o paciente estiver em uso de lítio adicionar valproato de sódio e vice-versa). Diante da falta de resposta, adicionar uma terceira medicação: outro estabilizador do humor em quem já está usando estabilizador + antipsicótico; em quem já está usando dois estabilizadores do humor, utilizar um antipsicótico. Nos casos com sintomatologia mais leve, não havendo resposta a um primeiro estabilizador do humor, é possível tentar trocar o estabilizador de humor antes de proceder associações. Nos casos mais graves, pode ser necessário o uso de dois estabilizadores do humor + um antipsicótico.

$3^{\circ}$ passo: casos de THB não-especificado ou com fenótipo amplo (irritabilidade marcada, mas sem episodicidade)

Não há consenso na literatura se esses casos representam THB verdadeiro ou se tratam de quadros de transtornos disruptivos do comportamento com sintomatologia marcada. Com base na evidência de resposta a antipsicóticos nos quadros de transtornos disruptivos em crianças e adolescentes, tem sido sugerido o uso inicial de antipsicóticos de segunda geração nesses quadros (para uma discussão mais aprofundada sobre o uso de antipsicóticos em crianças, veja Findling e McNamara, 


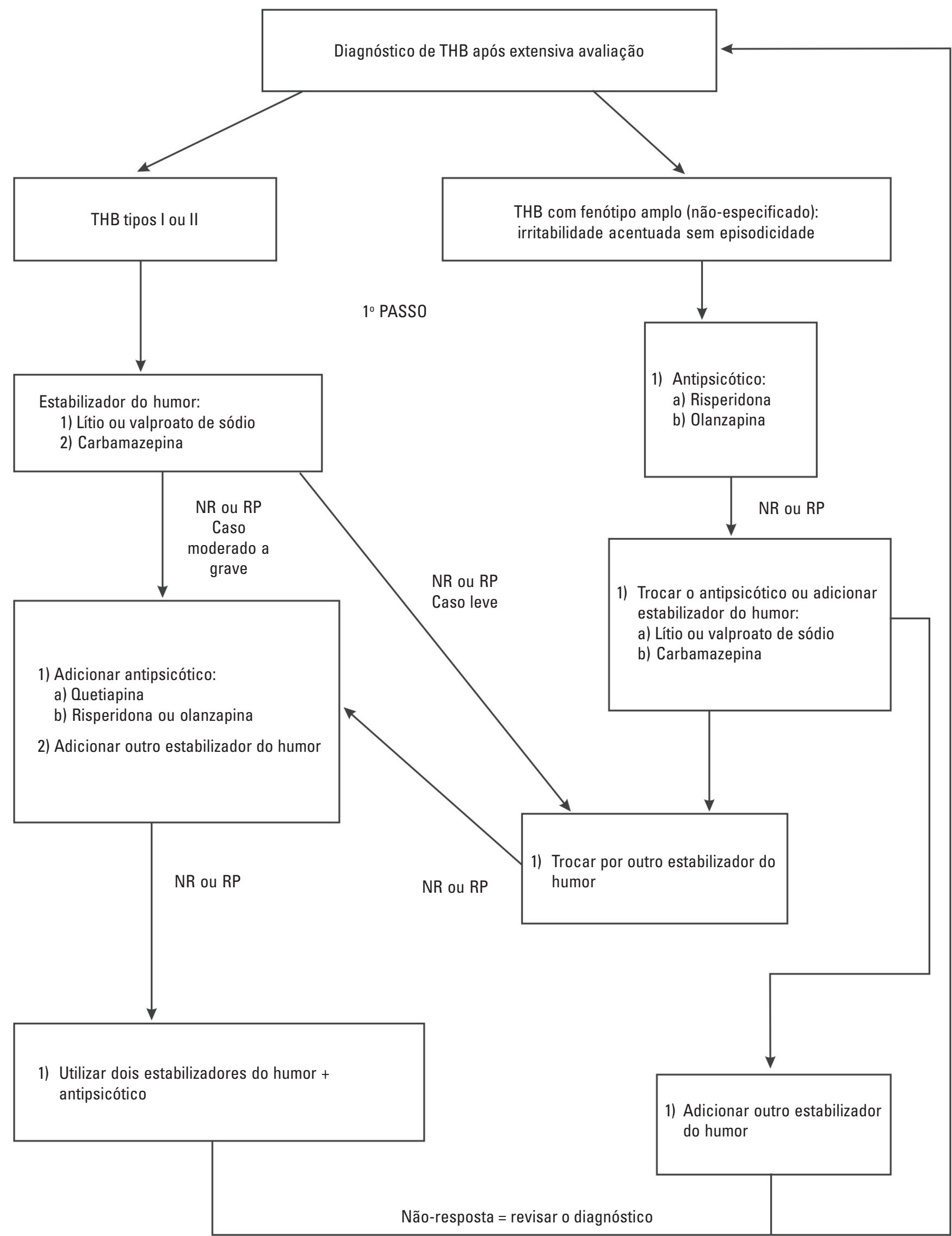

$\mathrm{NR}=$ não-resposta; $\mathrm{RP}=$ resposta parcial

Figura 1. Algoritmo para o tratamento farmacológico do THB em crianças e adolescentes. 
2004). Não existem estudos comparativos que indiquem a preferência por algum antipsicótico específico. Nãoresposta ou resposta parcial indicaria a troca para outro antipsicótico ou estabilizador do humor (lítio ou valproato de sódio). Persistência de não-resposta indicaria associação de um segundo estabilizador do humor.

\section{Comentários}

1. Na presença de sintomas psicóticos, está sempre indicado o uso associado de antipsicótico ao estabilizador do humor. Essa pode ser uma situação para início de uso de antipsicótico e adição de estabilizador de humor conforme resposta.

2. Presença de TDAH em comorbidade. Biederman et al. (1999) realizaram um estudo retrospectivo de análise de prontuários de 38 crianças e adolescentes com THB e TDAH. A chance de melhora do TDAH após estabilização dos sintomas maníacos foi 7,5 vezes maior do que aquela registrada antes da melhora dos sintomas maníacos. A recorrência de sintomas maníacos após sua inicial estabilização inibiu significativamente a resposta do TDAH à medicação. Assim, os algoritmos de tratamento têm proposto que a introdução de estimulantes seja feita nos casos comórbidos apenas após o uso de estabilizadores do humor e/ou de antipsicótico (Pavuluri et al., 2002; Pavuluri et al., 2004a). É importante salientar que, mesmo existindo uma alta comorbidade entre THB e TDAH em crianças e adolescentes, não foram encontrados estudos controlados na literatura que avaliem a melhor estratégia farmacológica para o tratamento das duas condições quando em comorbidade. Está em andamento, na nossa unidade de psicofarmacologia, protocolo aberto avaliando a eficácia do aripiprazole isolado e em associação ao metilfenidato nessa comorbidade. Por não parecer associado a ganho de peso e pelo possível mecanismo estabilizador da transmissão dopaminérgica, parece ser uma droga promissora nos quadros de THB em comorbidade com TDAH em crianças e adolescentes.

3. A questão da depressão bipolar. A única evidência disponível em crianças e adolescentes parece indicar que o uso de ISRS possa ser tentado nessa situação, após estabilização do THB com estabilizadores do humor (vide acima). Entretanto, essa é uma questão de pesquisa ainda aberta, sendo interessante que o clínico possa se valer da evidência disponível na literatura de adultos. Para uma revisão detalhada sobre depressão bipolar, veja artigo "Tratamento da depressão bipolar" (Lafer e Soares, 2005), neste suplemento.

4. Atenção aos eventos adversos. É fundamental que o clínico possa discutir com a família e a criança ou adolescente (usando linguagem apropriada) os possíveis eventos adversos da medicação. $\mathrm{O}$ registro de que essa discussão foi realizada e de que todos os potenciais eventos adversos foram apresentados deve constar no prontuário. A discussão de todos os potenciais eventos adversos de cada medicação usada para controle do THB em crianças foge ao alcance dessa revisão. Entretanto, vale lembrar que o clínico tem o dever de estar atento aos eventos adversos que são mais freqüentes nessa faixa etária (por exemplo, hepatotoxicidade ou ovários policísticos em meninas com o valproato de sódio). O aumento de peso acentuado é um fator limitante para o uso de algumas das medicações mencionadas para uso em crianças. Assim, está em andamento na nossa unidade de psicofarmacologia de crianças e adolescentes protocolo aberto avaliando a eficácia do topiramato isolado ou em associação com antipsicótico no controle de sintomas maníacos em crianças e adolescentes que já usaram outras medicações, mas tiveram aumento significativo de peso. Por fim, embora ainda muito pouco discutido na literatura sobre intervenções farmacológicas no THB em crianças, o adequado monitoramento do perfil metabólico dos pacientes deve sempre ser realizado.

5. Manutenção. Não existem estudos bem conduzidos metodologicamente avaliando a melhor estratégia para a manutenção do tratamento em crianças e adolescentes. A princípio, mantém-se o tratamento de maior eficácia no quadro agudo. A duração desse tratamento pode depender de questões individuais, como número e gravidade de episódios anteriores, ou dados da história familiar de resposta a fármacos no THB. Novamente, o clínico deverá se valer dos dados provenientes dos estudos em adultos. Para revisão detalhada sobre tratamento de manutenção, veja artigo "Tratamento do transtorno bipolar Eutimia” (Souza, 2005), neste suplemento).

\section{Conclusões}

O reconhecimento do THB em crianças e adolescentes tem aumentado significativamente nos últimos anos. Entretanto, é escassa a disponibilidade de evidência científica de qualidade para guiar o clínico na decisão do tratamento farmacológico a ser indicado para o transtorno em crianças e adolescentes. É importante salientar que, embora o tratamento farmacológico melhore o THB em crianças, a grande maioria dessas segue com sintomas residuais apesar do uso concomitante de várias medicações. Portanto, protocolos de intervenção psicossocial em associação com o uso de medicação devem ser elaborados para crianças e adolescentes com o transtorno. Nesse sentido, Pavuluri et al. (2004b) recentemente demonstraram a aceitabilidade e efetividade de uma intervenção cognitivocomportamental focada na família para o tratamento do THB em crianças e adolescentes. Por fim, estudos controlados precisam ser realizados avaliando a eficácia das medicações para o transtorno na presença de comorbidades, assim como adequada avaliação de eventos adversos associados às medicações usadas. 
Referências bibliográficas

Bhangoo, R.K.; Lowe, C.H.; Myers, F.S. et al. - Medication Use in Children and Adolescents Treated in the Community for Bipolar Disorder. J Child Adolesc Psychopharmacol 13: 515-522, 2003.

Biederman, J. - Open Trial of Atypical Neuroleptics in Preschoolers with Bipolar Disorder. Presented in the NIMH Pediatric Bipolar Disorder Conference Boston, 2004.

Biederman, J.; Mick, E.; Prince, J. et al. - Systematic Chart Review of the Pharmacologic Treatment of Comorbid Attention Deficit Hyperactivity Disorder in Youth with Bipolar Disorder. J Child Adolesc Psychopharmacol 9: 247-256, 1999.

BIEDERMAn, J.; Mick, E.; SPENCER, T.J. et al. - Therapeutic Dilemmas in the Pharmacotherapy of Bipolar Depression in the Young. J Child Adolesc Psychopharmacol 10: 185192, 2000.

Bouvard, M.P.; Bayle, F.; Dugas, M. - Open Trial of Carbamazepine in the Prevention of Recurrence of Bipolar Disorder in Adolescents. Encephale 19: 591600, 1993.

Carlson, G.A.; Jensen, P.S.; Finduing, R.L. et al. - Methodological Issues and Clinical Trials with Child and Adolescent Patients with Bipolar Disorder: Report of a Consensus Conference. J Child Adolesc Psychopharmacol 13: 115, 2003.

CARlSON, G.A.; LoneY, J.; SAlisBury, H. et al. - Stimulant Treatment In Young Boys with Symptoms Suggesting Childhood Mania: A Report from a Longitudinal Study. J Child Adolesc Psychopharmacol 10: 175-184, 2000.

Coyle, J.T.; Pine, D.S.; Charney, D.S. et al. and the Depression and Bipolar Support Alliance Consensus Development Panel. - Depression and Bipolar Support Alliance Consensus Statement on Unmeet Needs in Diagnosis and Treatment of Mood Disorders in Children and Adolescents. J Am Acad Child Adolesc Psichiatry 42: 1494-1503, 2003.

Davanzo, P.; Gunderson, B.; Belin, T. et al. - Mood Stabilizers in Hospitalized Children with Bipolar Disorder: a Retrospective Review. Psychiatry Clin Neurosci 57: 504510, 2003.

DelBello, M.P.; Kowatch, R.A.; Warner, J. et al. - Adjunctive Topiramate Treatment for Pediatric Bipolar Disorder: a Retrospective Chart Review. J Child Adolesc Psychopharmacol 12: 323-330, 2003.

DelBello, M.P.; Schwiers, M.L.; Rosenberg, H.L. et al. - A Doubleblind, Randomized, Placebo-controlled Study of Quetiapine as Adjunctive Treatment for Adolescent Mania. J Am Acad Child Adolesc Psychiatry 41: 12161223, 2002.

Finduing, R.L.; McNamara, N.K. - Atypical Antipsychotics in the Treatment of Children and Adolescents: Clinical Applications. J Clin Psychiatry 65 (Suppl 6): 30-44, 2004.
Findling, R.L.; McNamara, N.K.; Gracious, B.L. et al. Combination Lithium and Divalproex Sodium in Pediatric Bipolarity. J Am Acad Child Adolesc Psychiatry 42: 895901, 2003.

Frazier, J.A.; Biederman, J.; Tohen, M. et al. - A Prospective Open-label Treatment Trial of Olanzapine Monotherapy in Children and Adolescents with Bipolar Disorder. $J$ Child Adolesc Psychopharmacol 11: 239-250, 2001.

Frazier, J.A.; Meyer, M.C.; Biederman, J. et al. - Risperidone Treatment for Juvenile Bipolar Disorder: a Retrospective Chart Review. J Am Acad Child Adolesc Psychiatry 38: 960-965, 1999.

Geller, B.; Cooper, T.B.; Sun, K. et al. - Double-blind and Placebocontrolled Study of Lithium for Adolescent Bipolar Disorders with Secondary Substance Dependence. $J$ Am Acad Child Adolesc Psichiatry 37: 171-178, 1998.

Gracious, B.L.; Findling, R.L.; Seman, C. et al. - Elevated Thyrotropin in Bipolar Youths Prescribed Both Lithium and Divalproex Sodium. J Am Acad Child Adolesc Psychiatry 43: 215-220, 2004.

HenRY, C.A.; ZamVIL, L.S.; LAM, C. et al. - Long-term Outcome with Divalproex in Children and Adolescents with Bipolar Disorder. J Child Adolesc Psychopharmacol 13: 523-529, 2003.

Kafantaris, V.; Coletti, D.J.; Dicker, R. et al. - Adjunctive Antipsychotic Treatment of Adolescents with Bipolar Psychosis. J Am Acad Child Adolesc Psychiatry 40: 14481456, 2001.

Kafantaris, V.; Coletti, D.J.; Dicker, R. et al. - Lithium Treatment of Acute Mania in Adolescents: A Large Open Trial. $J$ Am Acad Child Adolesc Psychiatry 42: 1038-1045, 2003.

Kowatch, R.A.; Sethuraman, G.; Hume, J.H. et al. - Combination Pharmacotherapy in Children and Adolescents with Bipolar Disorder. Biol Psychiatry 53: 978-984, 2003.

Kowatch, R.A.; Suppes, T.; CARMOdY, T.J. et al. - Effect Size of Lithium, Divalproex Sodium, and Carbamazepine in Children and Adolescents with Bipolar Disorder. J Am Acad Child Adolesc Psychiatry 39: 713-720, 2000.

Leibenluft, E.; Charney, D.S.; Towbin, K.E. et al. - Defining Clinical Phenotypes of Juvenile Mania. Am J Psychiatry 160: 430-437, 2003.

LeWInSOHN, P.M.; KLeIN, D.N.; SeEley, J.R. - Bipolar Disorders in a Community Sample of Older Adolescents: Prevalence, Phenomenology, Comorbidity, and Course. J Am Acad Child Adolesc Psychiatry 34: 454-463, 1995.

Matos e SouzA, F.G. Tratamento de Transtorno Bipolar: Eutimia. Rev. Psiquiatr Clin 2005.

Pavuluri, M.N.; Henry, D.B.; Devineni, B. et al. - A Pharmacotherapy Algorithm for Stabilization And Maintenance of Pediatric Bipolar Disorder. J Am Acad Child Adolesc Psychiatry 43: 859-867, 2004a.

Pavuluri, M.N.; Graczyk, P.A.; Henry, D.B. - Child and Family Focused Cognitive-Behavioral Therapy for Pediatric 
Bipolar Disorder: Development and Preliminary Results. J Am Acad Child Adolesc Psychiatry 43: 528-537, 2004b.

Pavuluri, M.N.; Naylor, M.W.; JanicaK, P.G. et al. - Recognition and Treatment of Pediatric Bipolar Disorder. Contemporary Psychiatry 1: 1-10, 2002.

Soutullo, C.A.; DelBello, M.P.; Ochsner, J.E. et al. - Severity of Bipolarity in Hospitalized Manic Adolescents with History of Stimulant or Antidepressant Treatment. $J$ Affect Disord 70: 323-327, 2002.

Strober, M.; Deantonio, M.; Schmidt-Lackner, S. et al. - Early Childhood Attention Deficit Hyperactivity Disorder Predicts Poorer Response to Acute Lithium Therapy in Adolescent Mania. J Affect Disord 51: 145-151, 1998.

Strober, M.; Morrel, W.; Burroughs, J. et al. - A Family Study of Bipolar I Disorder in Adolescence: Early Onset of
Symptoms Linked to Increased Familial Loading and Lithium Resistance. J Affect Disord 15: 255-268, 1988.

Szoвот, C.; Rohde, L.A. - Lithium in Bipolar Adolescents with 4, Secondary Substance Dependency (letter). J Am Acad Child Adolesc Psychiatry 38: 1999.

Tramontina, S.; Schimitz, M.; Polanczyk, G. et al. - Juvenile Bipolar Disorder in Brasil : Clinical and Treatment Findings. Biological Psychiatry 53: 1043-1049, 2003.

Wagner, K.D.; Weller, E.B.; Carlson, G.A. et al. - An Openlabel Trial of Divalproex in Children and Adolescents with Bipolar Disorder. J Am Acad Child Adolesc Psychiatry 41: 1224-1230, 2002.

WeSt, S.; KecK, P.; McELroy, S. - Open Trial of Valproate in the Treatment of Adolescent Mania. J Child Adolesc Psychopharmacol 4: 263-267, 1994. 